\title{
CONTROLE DE QUALIDADE DE CÁPSULAS MANIPULADAS CONTENDO ATORVASTATINA CÁLCICA
}

Diego da Silva Ribeiro, Nayra Mendes da Silva, Ana Julia Pereira Santinho Gomes.

Universidade Federal de São João Del-Rei - UFSJ. MG. E-mail: ajpsant@ufsj.edu.br

\begin{abstract}
RESUMO
A atorvastatina cálcica (ATC) é um dos fármacos mais usados no tratamento da dislipidemia. A manipulação de cápsulas contendo ATC tem sido frequentemente solicitada por ser um medicamento de dispensação excepcional (uso contínuo e alto custo). A ATC apresenta baixa solubilidade e alta permeabilidade, portanto, adjuvantes farmacêuticos são capazes de interferir em sua solubilidade. $O$ objetivo deste trabalho foi avaliar a qualidade de cápsulas de ATC preparadas em farmácias de Divinópolis, Brasil. A espectroscopia de infravermelho com Transformada de Fourier (FTIR) foi empregada para identificação da ATC. Testes de determinação de peso (DP), uniformidade de conteúdo (UC) e dissolução também foram realizados. O FTIR confirmou a autenticidade da ATC. Todos os lotes foram aprovados em relação à DP, porém apenas $67 \%$ foram aprovados em relação à UC, embora tenham sido aprovados quanto ao valor de aceitação. Observou-se também que $1 / 3$ dos produtos não apresentou a taxa de dissolução especificada. Isto se deve à composição do excipiente impactando em um perfil de dissolução significativamente mais lento em comparação com os demais. Notou-se que a escolha de um excipiente específico para ATC juntamente com um procedimento de encapsulamento apropriado apresentam papéis fundamentais para garantir a qualidade de um medicamento de dispensação excepcional, cujo comportamento da dissolução pode influenciar a absorção oral. Em conclusão, demonstrou-se a importância do monitoramento do processo magistral ampliando a relevância da constante avaliação da qualidade de formas farmacêuticas trazendo à tona as dificuldades do setor magistral, bem como se evidenciou a efetividade dos critérios de aprovação empregados até o momento.
\end{abstract}

Palavras-chave: Dislipidemia, estatinas, técnicas in vitro, cápsulas, farmácias de manipulação.

\section{QUALITY CONTROL OF COMPOUNDED CAPSULES CONTAINING ATORVASTATIN CALCIUM}

\begin{abstract}
Atorvastatin calcium (ATC) is one of the most used drugs in the treatment of dyslipidemia. The compounding of capsules containing ATC has often been requested as it is an exceptional dispensing drug (continuous use and high cost). ATC presents low solubility and high permeability, therefore, pharmaceutical ingredients are capable of interfering with its solubility. The aim of this work was to evaluate the quality of ATC capsules prepared in compounding pharmacies in Divinópolis, Brazil. Fourier Transform Infrared spectroscopy (FTIR) was used to identify the ATC. Tests for weight determination (WD), content uniformity (CU) and dissolution were also carried out. The FTIR confirmed the authenticity of ATC. All batches were approved in relation to the WD, however only $67 \%$ were approved in relation to the CU, although all have been approved for acceptance value. It was also observed that $1 / 3$ of the products did not display the specified dissolution rate. This is due to the excipient composition impacting on a significantly slower dissolution profile compared to the others. It was noted that the choice of a specific excipient for ATC together with an appropriate encapsulation procedure play key roles in ensuring the quality of an exceptional dispensing drug, whose dissolution behavior can influence oral absorption. In conclusion, the importance of monitoring the compounding process was demonstrated, expanding the relevance of the constant evaluation of the quality of pharmaceutical dosage forms, bringing to light the
\end{abstract}


difficulties of the compounding sector, as well as the effectiveness of the approval criteria employed to date.

Keywords: Dyslipidemia, statins, in vitro techniques, capsules, compounding pharmacies.

\section{INTRODUÇÃO}

A terapia mais validada clinicamente para reduzir a incidência de eventos cardiovasculares emprega especialmente as reduções mais intensivas do colesterol da lipoproteína de baixa densidade (LDL-C) através do uso de estatinas ou inibidores da hidroximetilglutaril coenzima $A$ (HMG CoA) redutase. A depleção intracelular de colesterol estimula a liberação de fatores transcricionais e, consequentemente, a síntese e a expressão na membrana celular de receptores para captação do colesterol circulante, como o LDL-R ${ }^{1}$.

Assim, a ação das estatinas pode potencialmente influenciar todo o conjunto das lipoproteínas circulantes que interagem com o LDL-R, tais como a LDL, a VLDL e remanescentes de quilomícrons. Além disso, ao inibirem a HMGCoA redutase, as estatinas reduzem a formação de mevalonato e de radicais isoprenil, atenuando a ativação de proteínas fundamentais à resposta inflamatória e à biodisponibilidade de óxido nítrico. Adicionalmente, as estatinas reduzem os triglicérides também mediante $o$ aumento da expressão de LDL-R e, por conseguinte, pela remoção de lipoproteínas ricas em triglicérides do plasma ${ }^{1}$.

Atualmente, o tratamento farmacológico das dislipidemias conta com seis estatinas, sendo elas atorvastatina cálcica (ATC), fluvastatina sódica, pitavastatina cálcica, pravastatina sódica, rosuvastatina cálcica e sinvastatina ${ }^{1}$.

A ATC, medicamento antilipêmico de dispensação excepcional (uso contínuo e alto custo), desempenha um papel relevante no tratamento de pacientes que necessitam reduzir altos níveis de colesterol LDL-c $\mathrm{c}^{2,3}$.

Este fármaco apresenta-se como um pó cristalino branco muito levemente solúvel em água e levemente solúvel em tampão fosfato, $\mathrm{pH}$ 7,4, acetonitrila e etanol, e livremente solúvel em metanol. Apresenta polimorfismo, pKa igual a 4,46, cLogP de 4,46 e $\log P$ de 5,05. Sua permeabilidade é alta no ambiente intestinal em $\mathrm{pH}$ entre $6,0-6,5$, contudo, devido a sua baixa solubilidade em meio aquoso encontra-se na classe II (baixa solubilidade/alta permeabilidade) dentro do sistema de classificação biofarmacêutica ${ }^{4,5}$.

Há alguns anos, a expiração da patente da $\mathrm{ATC}^{6}$ reduziu o custo deste fármaco ocasionando aumento de sua prescrição para o gerenciamento da hipercolesterolemia e subseqüente tratamento da aterosclerose.

Em paralelo, verifica-se um aumento expressivo no numero de farmácias de manipulação ${ }^{7}$ juntamente com vários trabalhos realizados no intuito de discutir e assegurar a qualidade de medicamentos magistrais, principalmente formas farmacêuticas sólidas orais de liberação imediata (FFSOLI), tais como, cápsulas contendo fármaco da classe biofarmacêutica II, pois neste caso, a solubilidade e a taxa de dissolução tornam-se fatores críticos relacionados à biodisponibilidade oral ${ }^{8-11}$.

A ATC por ser um medicamento de uso contínuo e de grande importância para a saúde pública, assim como outras estatinas, está presente na Relação Nacional de Medicamentos Essenciais (RENAME) de 2019 elaborada a partir da Política Nacional de Medicamentos de 1998 e da Política Nacional de Assistência Farmacêutica de 2004 como um dos seus eixos estratégicos, bem como instrumento racionalizador da prescrição, da dispensação e do uso dos medicamentos ${ }^{3}$.

No município de Divinópolis-MG, constatou-se que a sinvastatina é prescrita mais frequentemente dentre os medicamentos usados para tratar dislipidemia em pacientes atendidos pelo SUS ${ }^{12}$. Em contrapartida, a dispensação de medicamentos por farmácias magistrais propicia adesão menos onerosa aos tratamentos farmacoterapêuticos ${ }^{9}$, como a ATC, estatina também prescrita, que representa um custo elevado ao usuário.

Considerando que formas farmacêuticas manipuladas devem atender as "Boas práticas de manipulação de preparações magistrais e oficinais para uso humano em farmácias"13,14, este trabalho teve como objetivo avaliar a qualidade de cápsulas de ATC preparadas em farmácias de Divinópolis-MG, um medicamento de uso contínuo e alto custo, cuja taxa de dissolução pode influenciar a absorção oral. 


\section{METODOLOGIA}

\section{Identificação da ATC}

Inicialmente, a ATC tri-hidratada utilizada pelas farmácias de manipulação situadas na cidade de Divinópolis-MG, denominadas Farmácias A, B e C, foi submetida à análise espectroscópica de infravermelho por transformada de Fourier (FTIR) usando um espectrofotômetro de infravermelho Shimadzu modelo IRAffinity-1. Para tanto, uma pequena quantidade de ATC foi homogeneizada com $\mathrm{KBr}$ previamente dessecado, compactada (10 toneladas) até formar discos transparentes com espessura de aproximadamente 1 milímetro que foram inseridos diretamente no espectrofotômetro. Os espectros de FTIR foram obtidos na faixa de 4000 a $500 \mathrm{~cm}^{-1}$, sob $4 \mathrm{~cm}^{-1}$ de resolução, à temperatura ambiente ( $25 \stackrel{\circ}{\circ})$. Em seguida, foram comparados com os registros disponíveis na literatura ${ }^{5,15}$.

\section{Preparação das cápsulas}

As cápsulas foram preparadas em lotes de 60 unidades $(n=3)$ contendo $10 \mathrm{mg}$ de ATC trihidratada $\left[\left(\mathrm{C}_{33} \mathrm{H}_{34} \mathrm{FN}_{2} \mathrm{O}_{5}\right)_{2} \mathrm{Ca}^{2+} \cdot 3 \mathrm{H}_{2} \mathrm{O}\right]$, cuja massa molecular é $1209,42 \mathrm{~g} / \mathrm{mol}$, calculada sobre a base anidra $(1155,34 \mathrm{~g} / \mathrm{mol})$ em acordância farmacopéica ${ }^{5,16}$, aplicando-se um fator de correção de 1,047. A Tabela 1 traz as ordens de manipulação disponibilizadas pelas farmácias.

A composição dos excipientes utilizados pelas farmácias encontra-se na Tabela 2.

Tabela 1. Insumos farmacêuticos presentes nas cápsulas de atorvastatina cálcica (ATC) preparadas pelas farmácias em Divinópolis-MG, Brasil.

\begin{tabular}{lcccccc}
\hline \multirow{2}{*}{$\begin{array}{l}\text { Insumo } \\
\text { farmacêutico }\end{array}$} & \multicolumn{2}{c}{ Farmácia A } & \multicolumn{2}{c}{ Farmácia B } & \multicolumn{2}{c}{ Farmácia C } \\
\cline { 2 - 7 } & $\begin{array}{c}\text { Massa } \\
(\mathrm{mg})\end{array}$ & $\begin{array}{c}\% \text { na } \\
\text { cápsula* }\end{array}$ & $\begin{array}{c}\text { Massa } \\
(\mathrm{mg})\end{array}$ & $\begin{array}{c}\% \text { na } \\
\text { cápsula* }\end{array}$ & $\begin{array}{c}\text { Massa } \\
(\mathrm{mg})\end{array}$ & $\begin{array}{c}\text { \% na } \\
\text { cápsula* }\end{array}$ \\
\hline $\begin{array}{l}\text { ATC tri-hidratada } \\
\text { Excipiente }\end{array}$ & 10,5 & 8,3 & 10,5 & 5,3 & 10,5 & 5,3 \\
$\begin{array}{l}\text { Cápsula } \mathrm{N}^{\circ} 4 \\
\text { Capacidade 0,21 cm }\end{array}$ & 75,7 & 91,7 & 137,7 & 94,7 & 138,2 & 94,7 \\
$\begin{array}{l}\text { Cápsula } \mathrm{N}^{\circ} \text { 3 } \\
\text { Capacidade 0,30 } \mathrm{cm}^{3}\end{array}$ & 40,0 & - & - & - & - & - \\
Massa teórica total & - & - & 48,0 & - & 49,0 & - \\
\hline
\end{tabular}

* Os cálculos percentuais foram realizados considerando a dose de $10,5 \mathrm{mg}$ de ATC tri-hidratada equivalente a $10 \mathrm{mg}$ de atorvastatina e densidade aparente após empacotamento de $0,60 \mathrm{~g} / \mathrm{cm}^{3}$.

Tabela 2. Composição do excipiente utilizado nas cápsulas de atorvastatina cálcica (ATC) fornecidas pelas farmácias em Divinópolis-MG, Brasil.

\begin{tabular}{lcccc}
\hline \multirow{2}{*}{ Substância adjuvante } & \multicolumn{3}{c}{ Farmácia } & \multirow{2}{*}{ Categoria funcional $^{17,18}$} \\
& A (\%) & B (\%) & C (\%) & Diluente \\
\hline Carbonato de cálcio & 30 & 15 & ND & Lubrificante \\
Estearato de magnésio & 1 & 1 & ND & Diluente \\
Dióxido de silício coloidal & 1 & 2 & ND & Absorvente/Ativador de fluxo \\
Celulose microcristalina & 8 & 41 & ND & Diluente altamente hidrofílico \\
Lactose mono-hidratada & 60 & 41 & ND & Diluente
\end{tabular}

ND - porcentagem não declarada.

Em seguida, avaliou-se a qualidade dos produtos aplicando-se o ensaio de determinação de peso em cápsulas obtidas pelo processo magistral $^{19}$ e os ensaios de uniformidade de conteúdo (UC) e de dissolução ${ }^{16}$.

\section{Determinação de peso}

O teste de determinação de peso aplicase a formas farmacêuticas sólidas em dose unitária, como é o caso de cápsulas duras usadas na Farmácia de Manipulação ${ }^{19}$. Neste caso, três parâmetros foram considerados para aprovação: 1) Peso médio $\left(P_{\text {médio }}\right)$ das cápsulas e respectivo 
limite de variação (LV); 2) Desvio padrão relativo (DPR); e 3) Variação do conteúdo teórico (VCT) das cápsulas, como segue:

Peso médio das cápsulas. Foram pesadas, individualmente, dez unidades de cápsulas manipuladas íntegras usando Balança analítica Marte, modelo AY220. Em seguida, foi calculado o $P_{\text {médio }}$ que deve estar compreendido dentro de um LV de $\pm 10 \%$ para cápsulas duras com valor inferior a $300 \mathrm{mg}^{19,20}$.

Desvio padrão relativo (DPR). O DPR foi calculado aplicando-se a seguinte equação: $D P R=$ $\left(D P / P_{\text {médio }}\right) \times 100$, em que $P_{\text {médio }}$ é o peso médio das cápsulas e DP é o desvio padrão do $P_{\text {médio }}{ }^{19}$.

Variação do conteúdo teórico (VCT). Dáse determinando a quantidade teórica mínima de pó $\left(Q_{\text {teor.min. }}\right)$ e a quantidade teórica máxima de pó $\left(Q_{\text {teor.max. }}\right)$ de acordo com os extremos de pesos obtidos na pesagem das cápsulas. Para tanto, ao determinar-se o $P_{\text {médio, foram }}$ destacados os pesos da cápsula mais leve $\left(P_{\text {cápsula }}\right.$ mais leve) e o da mais pesada ( $\left.P_{\text {cápsula mais pesada }}\right)$, a partir dos quais aplicaram-se as equações a seguir $^{19}$ :

$$
\begin{gathered}
Q_{\text {teor.mín. }}=\frac{P_{\text {cápsula mais leve }}}{M T} \times 100 \\
Q_{\text {teor.max. }}=\frac{P_{\text {cápsula mais pesada }}}{M T} \times 100
\end{gathered}
$$

Em que MT = massa teórica total descrita por cada farmácia (Tabela 1 ).

\section{Uniformidade de conteúdo (UC)}

O método de UC para preparações em doses unitárias baseia-se no doseamento do conteúdo individual do fármaco a partir de um número de doses unitárias a fim de determinar se o conteúdo individual está dentro dos limites especificados ${ }^{20}$.

Para determinação da UC selecionaramse trinta unidades, dentre as quais dez unidades foram submetidas individualmente ao procedimento para UC, calculando-se posteriormente $O$ valor de aceitação (VA) segundo a equação $V A=[M-X]+k . s$, em que: $M$ $=$ número de unidades testadas; $X$ é a média dos conteúdos individuais expressa como porcentagem da quantidade declarada; $\mathrm{k}=2,4$ (constante de aceitabilidade para $\mathrm{n}=10$ ); e $\mathrm{s}=$ desvio padrão da amostra. Após o cálculo acima, o resultado é dado como $L 1$ e deve ser inferior a 15.
A ATC presente nas cápsulas foi quantificada por espectroscopia de absorção na região do ultravioleta (UV) usando um espectrofotômetro de UV-VIS Nova Instruments, modelo 1600UV. Em paralelo, também foi avaliada a influência dos excipientes na leitura espectrofotométrica das amostras.

Seleção do comprimento de onda máximo $\left(\lambda_{\max }\right)$. Foi realizada através de varredura espectral na região do UV $(190-390 \mathrm{~nm})$, com intervalos de $2 \mathrm{~nm}$. Posteriormente, foi realizado nova varredura na faixa compreendida entre 230 a $250 \mathrm{~nm}$, com intervalos de $1 \mathrm{~nm}$, para definir o $\lambda_{\max }$ para a ATC em solução metanólica preparada pesando-se acuradamente, $100 \mathrm{mg}$ de ATC, que foi transferida para um balão volumétrico de 100 $\mathrm{mL}(1 \mathrm{mg} / \mathrm{mL})$. Em seguida, uma alíquota de 250 $\mu \mathrm{L}$ foi transferida para um balão volumétrico de $25 \mathrm{~mL}$, o qual foi completado com metanol dando origem a uma solução $10 \mu \mathrm{g} / \mathrm{mL}$ de ATC usada para leitura espectrofotométrica usando cubeta de quartzo de $1 \mathrm{~cm}$.

Preparação da curva analítica I. Foi confeccionada uma curva analítica de ATC, padrão analítico (P.A.), em metanol a partir de uma solução estoque contendo $1 \mathrm{mg} / \mathrm{mL}$ nas concentrações de $2,4,6,8,10$ e $12 \mu \mathrm{g} / \mathrm{mL}$, em triplicata, usando metanol como branco e $\lambda_{\max }$ de $241 \mathrm{~nm}$, obtendo-se $\mathrm{y}=0,044 \mathrm{x}+0,0022$ como equação da reta com coeficiente de correlação linear de Pearson $(r)$ de 0,9976 usada para determinar a quantidade, em $\mathrm{mg}$, e a respectiva porcentagem de ATC presente nas cápsulas, bem como o VA.

A UC nas amostras foi realizada abrindose cuidadosamente cada cápsula $(n=10)$ e dissolvendo o conteúdo da mesma com metanol, completando o volume em um balão volumétrico de $100 \mathrm{~mL}$, obtendo-se uma suspensão de cor branca na concentração de $100 \mu \mathrm{g} / \mathrm{mL}$. Em seguida, o conteúdo do balão foi filtrado usando papel de filtro pregueado. Procedeu-se, a diluição em metanol até obter uma solução de concentração estimada em $10 \mu \mathrm{g} / \mathrm{mL}$. Por fim, as leituras espectrofotométricas foram realizadas em $241 \mathrm{~nm}$ usando metanol como branco.

\section{Dissolução}

A dissolução das cápsulas de ATC foi realizada em hexaplicata usando um Dissolutor Ethiktechnology modelo 299. Para tanto, as cubas do dissolutor foram preenchidas com $900 \mathrm{~mL}$ de tampão fosfato $0,05 \mathrm{~mol} / \mathrm{L}, \mathrm{pH} 6,8$, usando a cesta como aparato de dissolução, sob 
temperatura de $37 \pm 0,5^{\circ} \mathrm{C}$ e $75 \mathrm{rpm}^{16}$. O perfil de dissolução foi obtido a partir da coleta de alíquotas de $4 \mathrm{~mL}$ nos intervalos de 5, 10, 15 e 30 minutos $^{21,22}$, repondo-se o tampão para manter o volume do meio de dissolução.

Concomitantemente foi avaliada a influência dos excipientes na leitura espectrofotométrica das amostras.

Seleção do $\boldsymbol{\lambda}_{\max }$. Foi realizada através de varredura espectral na região do UV (190 - 390 $\mathrm{nm}$ ), com intervalos de $2 \mathrm{~nm}$. Posteriormente, foi realizado nova varredura na faixa compreendida entre 240 a $250 \mathrm{~nm}$, com intervalos de $1 \mathrm{~nm}$, para definir o $\lambda_{\max }$ para a ATC em tampão fosfato 0,05 $\mathrm{mol} / \mathrm{L}, \quad \mathrm{pH} 6,8$, preparada pesando-se acuradamente, $100 \mathrm{mg}$ de ATC, que foi transferida para um balão volumétrico de $100 \mathrm{~mL}$ (1 mg/mL). Em seguida, uma alíquota de $250 \mu \mathrm{L}$ foi transferida para um balão volumétrico de 25 $\mathrm{mL}$, cujo volume foi completado com tampão fosfato $0,05 \mathrm{~mol} / \mathrm{L}, \mathrm{pH} 6,8$ dando origem a uma solução de ATC de $10 \mu \mathrm{g} / \mathrm{mL}$ usada para leitura espectrofotométrica usando cubeta de quartzo de $1 \mathrm{~cm}$.

Preparação da curva analítica II. Foi confeccionada uma curva de ATC, P.A., em tampão fosfato $0,05 \mathrm{~mol} / \mathrm{L}, \mathrm{pH} 6,8$, a partir de uma solução estoque contendo $1 \mathrm{mg} / \mathrm{mL}$ nas concentrações de $4,6,8,10,12,16$ e $20 \mu \mathrm{g} / \mathrm{mL}$, em triplicata, usando tampão fosfato $0,05 \mathrm{~mol} / \mathrm{L}$,
$\mathrm{pH}$ 6,8 como branco e $\lambda_{\max }$ de $241 \mathrm{~nm}$, obtendose $y=0,0363 x+0,0294$ como equação da reta com coeficiente de correlação linear de Pearson (r) de 09997 usada para determinar a quantidade, em $\mathrm{mg}$, e porcentagem de ATC dissolvida das cápsulas.

A quantidade de ATC liberada no meio de dissolução foi determinada após filtração das alíquotas coletadas nos tempos predeterminados. As leituras espectrofotométricas foram realizadas em 241 $\mathrm{nm}$ usando tampão fosfato $0,05 \mathrm{~mol} / \mathrm{L}, \mathrm{pH} 6,8$ como branco.

\section{Análise estatística}

Os resultados estão apresentados como média aritmética dos valores e desvio padrão da média $(X \pm S D)$. Comparações estatísticas foram realizadas usando $O$ teste de normalidade Shapiro-Wilk e ANOVA com $p<0,05$ como nível mínimo de significância.

\section{RESULTADOS}

A partir dos espectros de FTIR foi elaborada a Tabela 3 que traz os principais comprimentos de onda encontrados nas amostras de ATC utilizadas pelas Farmácias A, B e $C$ em comparação com aqueles descritos na literatura ${ }^{15}$.

Tabela 3. Comparação entre os comprimentos de onda $\left(\mathrm{cm}^{-1}\right)$ característicos observados nos espectros de FTIR de atorvastatina cálcica (ATC) descrito na literatura ${ }^{15}$ e de amostras de ATC disponíveis nas farmácias em Divinópolis-MG, Brasil.

\begin{tabular}{cccc}
\hline \multirow{2}{*}{$\begin{array}{c}\text { Comprimento de } \\
\text { onda }\left(\mathbf{c m}^{-1}\right)^{15}\end{array}$} & \multicolumn{3}{c}{ Comprimento de onda $\left(\mathbf{c m}^{-1}\right)$ da ATC } \\
\cline { 2 - 4 } & Farmácia A & Farmácia B & Farmácia C \\
\hline 3403,27 & 3400,50 & 3391,76 & 3363,66 \\
2955,15 & 2962,66 & 2969,95 & 3065,24 \\
1656,97 & 1656,95 & 1667,96 & 1651,07 \\
1564,97 & 1562,34 & 1557,77 & 1577,77 \\
1313,56 & 1313,52 & 1314,04 & 1317,36 \\
1104,39 & 1111,00 & 1107,54 & 1109,22 \\
751,62 & 752,24 & 749,43 & 746,45 \\
\hline
\end{tabular}

Parâmetros relacionados à determinação de peso ( $P_{\text {médio, }}$ LV, DPR e VCT) devem ser avaliados pelo farmacêutico para aprovar ou não as cápsulas para dispensação e compõem o Controle de Qualidade dos Medicamentos Manipulados (item 10.1 do roteiro de inspeção) ${ }^{13}$.

De acordo com a Tabela 4, nota-se que o LV de $\pm 10 \%$ para cápsulas duras com $\mathrm{P}_{\text {médio }}$ inferior a $300 \mathrm{mg}$ foi respeitado, além de exibirem DPR abaixo de $4 \%$ e VCT entre $90-110$ $\%$. Tais resultados são usados para verificar se as unidades de um mesmo lote apresentam uniformidade de peso ${ }^{19}$ e revelam que todos os lotes analisados foram aprovados em relação aos ensaios de determinação de peso, além disto, não foram observadas diferenças significativas entre os lotes preparados. 
Tabela 4. Valores encontrados para os parâmetros de determinação de peso e uniformidade de conteúdo de cápsulas de atorvastatina cálcica (ATC) preparadas por farmácias em Divinópolis-MG, Brasil.

\begin{tabular}{cccccccc}
\hline & \multicolumn{7}{c}{ Parâmetros } \\
\cline { 2 - 8 } $\begin{array}{c}\text { Farmácia } \\
\text { Lote }\end{array}$ & $\begin{array}{c}\mathbf{P}_{\text {médio }} \\
\mathbf{( m g )}\end{array}$ & $\begin{array}{c}\text { DV } \\
\mathbf{( m g )}\end{array}$ & $\begin{array}{c}\text { DPR } \\
\mathbf{( \% )}\end{array}$ & $\begin{array}{c}\text { VCT } \\
\mathbf{( \% )}\end{array}$ & $\begin{array}{c}\text { Teor de } \\
\text { ATC } \\
\text { (mg) }\end{array}$ & $\begin{array}{c}\text { Quantidade } \\
\text { declarada de } \\
\text { ATC (\%) }\end{array}$ & VA \\
\hline A1 & $126,1 \pm 1,7$ & $113,5-138,7$ & 1,4 & $97,1-105,5$ & $10,5 \pm 1,2$ & $105,2^{*}$ & 6,6 \\
A2 & $125,7 \pm 1,7$ & $113,1-138,3$ & 1,4 & $97,9-100,8$ & $10,5 \pm 0,4$ & 105,0 & 4,6 \\
A3 & $126,7 \pm 2,2$ & $114,0-139,4$ & 1,7 & $96,8-104,8$ & $10,1 \pm 0,5$ & 100,8 & 1,1 \\
B1 & $194,4 \pm 3,7$ & $175,0-213,8$ & 1,9 & $93,2-103,1$ & $9,3 \pm 0,3$ & $92,7 *$ & 6,5 \\
B2 & $196,8 \pm 3,8$ & $177,1-216,5$ & 1,9 & $94,9-104,1$ & $9,7 \pm 0,3$ & 97,0 & 2,1 \\
B3 & $197,2 \pm 3,6$ & $177,5-216,9$ & 1,8 & $95,7-104,4$ & $9,8 \pm 1,1$ & 98,4 & 2,7 \\
C1 & $196,8 \pm 6,3$ & $177,1-216,5$ & 3,2 & $92,2-107,0$ & $10,5 \pm 0,3$ & $105,2 *$ & 4,5 \\
C2 & $199,5 \pm 5,1$ & $179,6-219,5$ & 2,5 & $93,5-104,4$ & $10,1 \pm 1,4$ & 100,8 & 3,3 \\
C3 & $196,6 \pm 5,5$ & $176,9-216,3$ & 2,8 & $94,0-104,7$ & $10,2 \pm 1,7$ & 102,2 & 4,8 \\
\hline
\end{tabular}

*Valores fora da faixa especificada para comprimidos de ATC $(94,5 \%-105,0 \%)^{16}$. P $_{\text {médio }}=$ peso médio, $\mathrm{LV}=$ limite de variação, $\mathrm{DPR}=$ desvio padrão relativo, $\mathrm{VCT}$ = variação do conteúdo teórico, VA = valor de aceitação.

Já os resultados de UC revelaram que a quantidade de ATC declarada, em \%, apresentou valores fora do intervalo especificado em $1 / 3$ dos lotes analisados (A1, B1 e C1), envolvendo todas as farmácias do presente trabalho (Tabela 4), pois deveriam conter no mínimo $94,5 \%$ e no máximo $105,0 \%$ da quantidade declarada de atorvastatina $^{16}$. Embora os valores de aceitação encontrados tenham sido menores que $L 1$, apenas 2/3 (67 \%) dos lotes submetidos ao teste de UC foram aprovados (Tabela 4$)^{20}$.

Soma-se a isso, os ensaios de dissolução in vitro para FFSOLI tais como comprimidos e cápsulas, que são utilizados para garantir a qualidade lote a lote, orientar o desenvolvimento de novas formulações e assegurar a uniformidade da qualidade e do desempenho do medicamento ${ }^{22}$.

No caso da ATC, a tolerância (Q) é de 80 $\%$ da quantidade de fármaco, expressa em \% da quantidade declarada dissolvida em 15 minutos $^{16}$. Se cada unidade apresentar resultado igual ou maior do que $Q+5 \%$, o produto apresentará conformidade com o especificado ${ }^{20,22}$.

Os resultados de UC e de dissolução foram obtidos em $241 \mathrm{~nm}$ destacando-se a ausência de interferência dos excipientes.

Nas Figuras 1 e 2 pode-se observar, respectivamente, a taxa de ATC dissolvida em 15 minutos e o perfil de dissolução destas cápsulas.

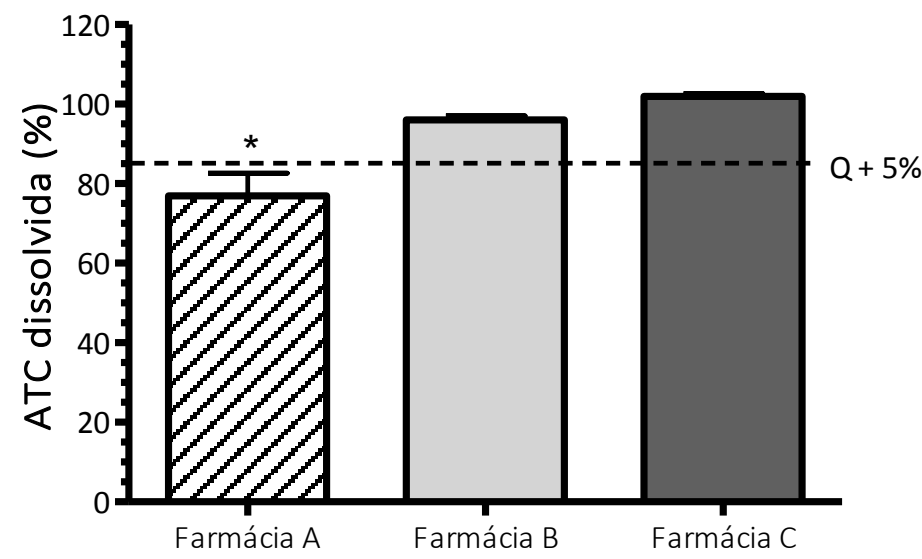

Figura 1. Taxa de atorvastatina cálcica (ATC) dissolvida em 15 minutos com tolerância (Q) de $80 \%+5 \%$, a partir de cápsulas preparadas por farmácias em Divinópolis-MG, Brasil, sendo * $p<0,05$. 


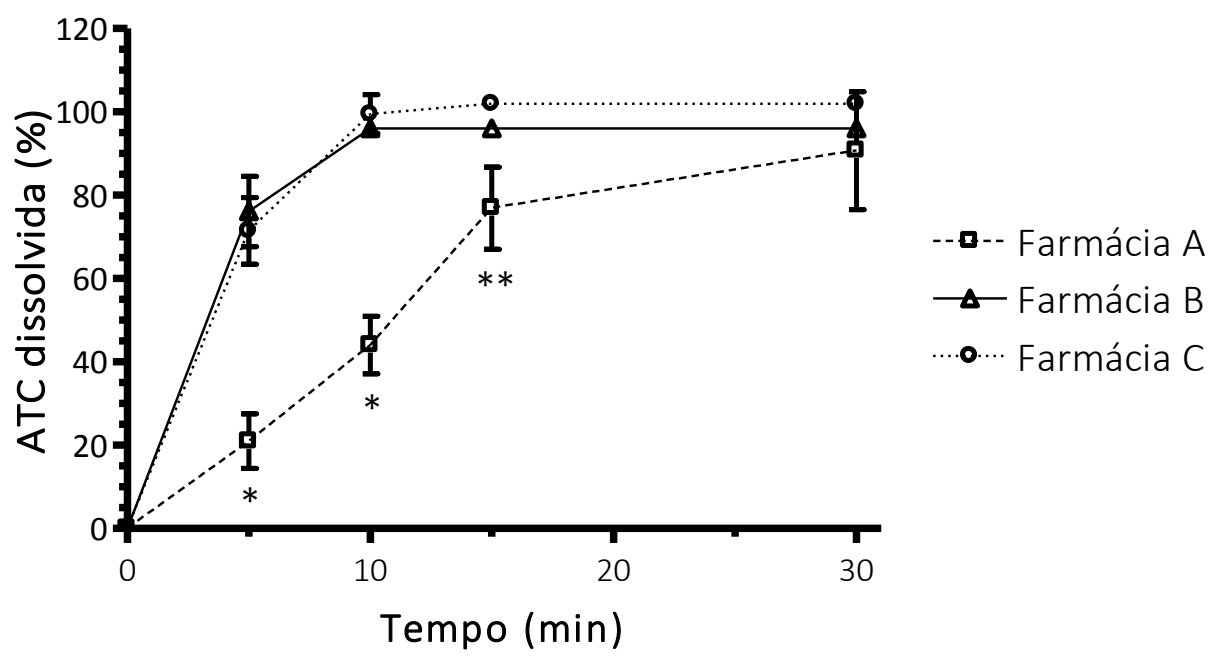

Figura 2. Perfil de dissolução de cápsulas de atorvastatina cálcica (ATC) preparadas por farmácias em Divinópolis-MG, Brasil, sendo * $p<0,001$ e ** $p<0,05$.

\section{DISCUSSÃO}

Os testes espectrofotométricos contribuem significativamente com relação à identificação de várias substâncias químicas. A absorção da radiação infravermelha é uma propriedade bastante característica de uma substância e é amplamente usada em propostas de identificação ${ }^{23}$, pois a partir da comparação do perfil espectral pode fornecer evidências conclusivas sobre a identidade da substância ${ }^{16}$.

Pode-se observar que as bandas encontradas nos espectros das amostras analisadas (Tabela 3) apresentaram similaridade com as descritas na literatura ${ }^{15}$. Contudo, apesar de serem próximas, não são idênticas. Isto se deve, provavelmente, a presença de água na molécula de ATC, além da espessura da amostra (fator que deve ser controlado quer no modo de transmitância usando um comprimento óptico fixo, bem como no modo de refletância, em que se deve controlar a profundidade de penetração da luz, para que não altere o espectro se a espessura da amostra aumenta).

As vibrações na faixa aproximada de absorção de $3403 \mathrm{~cm}^{-1}$ (Tabela 3) podem ser atribuídas a três causas ${ }^{24}$. Primeiramente, a deformação axial de $\mathrm{N}-\mathrm{H}$ de amida secundária $\left(3500-3400 \mathrm{~cm}^{-1}\right)$. Segundo, a deformação axial de $\mathrm{N}-\mathrm{H}$ de um composto heteroaromático $\left(\sim 3400 \mathrm{~cm}^{-1}\right)$. Por último, a deformação axial de $\mathrm{O}-\mathrm{H}$ de alcoóis $\left(3550-3200 \mathrm{~cm}^{-1}\right)$, corroborando com Narasaiah et al. ${ }^{15}$ que apontaram esta vibração como um estiramento $\mathrm{N}-\mathrm{H}$.
Na faixa aproximada de absorção de 2955 $\mathrm{cm}^{-1}$ (Tabela 3 ) podem ser atribuídas outras três causas. Primeiramente, a deformação axial de $\mathrm{C}-\mathrm{H}$ de metila e metileno $\left(3000-2840 \mathrm{~cm}^{-1}\right)$. Segundo, a deformação axial de $\mathrm{C}-\mathrm{H}$ de um composto heteroaromático $\left(3077-3003 \mathrm{~cm}^{-1}\right)$. Terceiro, a deformação axial de $=\mathrm{C}-\mathrm{H}$ de um composto aromático $\left(3100-3000 \mathrm{~cm}^{-1}\right)^{23,24,25}$, corroborando novamente com Narasaiah et al. $^{15}$ que apontaram esta vibração como um estiramento $\mathrm{C}-\mathrm{H}$.

Já o comprimento de onda próximo a $1656 \mathrm{~cm}^{-1}$ (Tabela 3 ) pode ser atribuído à deformação axial de $\mathrm{C}=\mathrm{O}$ de banda de amida I $\left(\sim 1650 \mathrm{~cm}^{-1}\right)$, e provavelmente a ligação de água $\left(1750-1500 \mathrm{~cm}^{-1}\right)$, pois se trata de uma amostra de ATC tri-hidratada ${ }^{23,24}$.

Nas faixas aproximadas de absorção de $1564 \mathrm{~cm}^{-1}$ e $1313 \mathrm{~cm}^{-1}$ (Tabela 3) tais comprimentos de onda podem ser atribuídos, respectivamente, a deformação axial do ânion carboxilado $\left(1650-1550 \mathrm{~cm}^{-1}\right)$ e a deformação axial de $\mathrm{C}-\mathrm{N}$ de amina aromática terciária (1360 $1310 \mathrm{~cm}^{-1}$ ), corroborando com Narasaiah et al. ${ }^{15}$ que apontaram esta vibração como um estiramento $\mathrm{C}-\mathrm{N}$, além da deformação axial das ligações do anel heteroaromático (1600 - 1300 $\left.\mathrm{cm}^{-1}\right)^{24}$.

Ademais, as faixas de absorção próximas de $1104 \mathrm{~cm}^{-1}$ e $751 \mathrm{~cm}^{-1}$ (Tabela 3) podem ser atribuídas, respectivamente, a deformação axial de $\mathrm{C}-\mathrm{O}$ de alcoóis $\left(1260-1000 \mathrm{~cm}^{-1}\right)$ e a deformação angular fora do plano de $=\mathrm{C}-\mathrm{H}$ de 
anéis aromático e heteroaromático (900 - 675 $\left.\mathrm{cm}^{-1}\right)^{24,25}$.

Por fim, a vibração correspondente à deformação de C-F presente na ATC é difícil de determinar. Isto porque a absorção $\mathrm{C}-\mathrm{X}$ ocorre em frequências muito baixas em que aparece uma variedade de outras bandas (impressão digital). Neste caso, a faixa de absorção correspondente à vibração de fluoreto se encontra entre 1400 e $1000 \mathrm{~cm}^{-125}$.

Com base nos dados acima, confirmou-se a identidade da ATC utilizada para compor as cápsulas manipuladas pelas Farmácias A, B e C envolvidas no presente trabalho.

Adicionalmente, sabe-se que as farmácias estão sujeitas a inspeção sanitária para verificação do cumprimento das Boas Práticas de Manipulação. Dentre outros itens citados na legislação tem-se o monitoramento do processo magistral, em que devem ser realizadas análises bimestrais de pelo menos uma fórmula, de teor e UC do fármaco em fórmulas, cuja unidade farmacotécnica contenha fármaco em quantidade $\leq 25 \mathrm{mg}^{8,14}$.

Assim, para assegurar a administração de doses corretas, cada unidade do lote de um medicamento deve conter quantidade de fármaco próxima da quantidade declarada. Neste sentido, o método de UC é exigido para cápsulas duras em que a dose e a proporção do fármaco sejam, respectivamente, inferiores a $25 \mathrm{mg}$ ou 25 $\%^{20}$, condições em que se enquadram as cápsulas de ATC do presente trabalho.

Ressalta-se que aprovação de todos os lotes em relação à determinação de peso é usada pelas farmácias para indicar que a massa de pós encapsulada está homogênea ${ }^{19}$. Entretanto, observa-se que este parâmetro não refletiu veridicamente a quantidade declarada de ATC, em \%, como evidenciado pelo ensaio de UC (Tabela 4). Tais informações corroboram com Baracat e colaboradores ${ }^{9}$.

A Figura 1 mostra que as cápsulas preparadas pela Farmácia A apresentaram taxa de dissolução significativamente inferior em comparação com as demais (Farmácias B e C). A tolerância permitida, após 15 minutos de dissolução, é de $85 \%$ em relação à dose declarada $^{16,20}$, portanto, pode-se dizer que este produto foi reprovado. Outros autores também descreveram resultados desta natureza ${ }^{9,10}$.

É válido comentar que o processo de dissolução de um fármaco a partir de uma FFSOLI envolve vários passos físico-químicos intermediários, tais como, umedecimento, intumescimento, capilaridade, solubilidade e difusão ${ }^{26}$. Desta maneira, a taxa e a extensão de dissolução podem ser afetadas por vários fatores, entre eles está a porosidade que pode permitir, ou não, o acesso do meio de dissolução à mistura pulvérea e a posterior difusão das moléculas de soluto dissolvidas para fora ${ }^{17,26}$.

Considerando que cápsulas duras são tipicamente preenchidas com fármaco(s) e substâncias adjuvantes necessárias para a estabilidade física e química da forma farmacêutica, torna-se relevante atentar para a escolha dos componentes que juntamente com o processo de produção podem afetar a cedência (dissolução) do fármaco ${ }^{26,27}$.

Sabe-se, entretanto que o preenchimento de cápsulas com a quantidade correta de pós é um importante parâmetro de qualidade e as propriedades de fluxo de misturas físicas finais, tais como ângulo de repouso, densidade aparente e porcentagem de compressibilidade, podem ser significativamente correlacionadas com o peso final e com a variabilidade de peso das cápsulas prontas ${ }^{28}$.

A Figura 2 mostra que as cápsulas de ATC preparadas pela Farmácia A apresentaram um perfil de dissolução significativamente mais lento nos primeiros 15 minutos em comparação com as cápsulas preparadas pelas Farmácias B e C. Isto pode ser atribuído a dois fatores, sendo eles: 1) A composição do excipiente usado pela Farmácia $\mathrm{A}$ (Tabela 2) constituído por $30 \%$ de $\mathrm{CaCO}_{3}$ em relação à Farmácia $\mathrm{B}$ (15\%), lembrando que a Farmácia $C$ não declarou as concentrações usadas, e 2) O procedimento de encapsulamento empregado.

Para explicar o primeiro dos fatores acima, deve-se considerar a alcalinidade do $\mathrm{CaCO}_{3}$, que apresenta $\mathrm{pH}$ 9,0 em dispersão aquosa a $10 \% \mathrm{~m} / \mathrm{V}^{18}$. Esta característica justifica seu emprego como diluente favorável à dissolução da ATC devido à formação de um microambiente capaz de evitar a lactonização deste fármaco e, consequente, formação de sal insolúvel ${ }^{2}$. Contudo, o $\mathrm{CaCO}_{3}$ apresenta densidade bruta $\left(D_{0}\right)$ de $0,8 \mathrm{~g} / \mathrm{cm}^{3}$ e densidade após empacotamento $\left(D_{f}\right)$ de $1,2 \mathrm{~g} / \mathrm{cm}^{3}$. Portanto, o índice de Hausner $\left(D_{f} / D_{0}\right)$ resulta em 1,5 caracterizando-o como de fluxo restrito e a porcentagem de compressibilidade $\left(D_{f}-D_{o}\right) / D_{f}$. 100) resulta em $33 \%$, apontando-o como uma substância que pode prejudicar o escoamento das partículas pulvéreas em função de sua 
elevada coesividade ${ }^{17,18,29}$. Compreende-se aqui que a Farmácia $A$ usou uma mistura de pós com fluidez inferior em relação às demais, fato que interferiu negativamente no procedimento de encapsulamento ${ }^{17}$.

Em se tratando do segundo fator, deve-se considerar também a capacidade volumétrica da cápsula usada pela Farmácia A (Tabela 1) que contribuiu para ampliar a dificuldade do processo, pois o diâmetro do orifício para o escoamento dos pós é o menor (cápsula № $4=$ $0,21 \mathrm{~mL}$ ). Sugere-se que o empacotamento foi promovido através do uso de socador, acessório auxiliar no encapsulamento de pós com restrição de fluxo ${ }^{30}$.

Embora o emprego deste tipo de acessório leve frequentemente a valores de DPR elevados $^{30}$, isto não foi observado no presente trabalho (Tabela 4), porém o uso do socador pode causar aproximação das partículas, e consequente redução da porosidade. Isso pode aumentar tanto $\mathrm{o}$ número de contatos interparticulares quanto as superfícies de contato, elevando ainda mais as forças de coesão ${ }^{17}$.

Sugere-se, portanto que a mistura contida nas cápsulas e o procedimento de encapsulamento adotado pela Farmácia A apresentou implicação no perfil de dissolução em questão.

Diante deste contexto, recomenda-se que a Farmácia $\mathrm{A}$ diminua a concentração de $\mathrm{CaCO}_{3}$ no excipiente a ser misturado com ATC para minimizar o efeito de tal substância tanto na fluidez quanto na dissolução destas cápsulas. A propósito, Souza e colaboradores ${ }^{8}$ prepararam cápsulas de nifedipina e demonstraram que a escolha dos excipientes exibiu papel decisivo na taxa de dissolução deste fármaco.

Em adição, tanto a taxa quanto a extensão da dissolução alcançadas pelas cápsulas da Farmácia B (Figuras 1 e 2, respectivamente) podem ser atribuídas à elevada porcentagem de lactose (41\%), diluente altamente hidrofílico, capaz de: i) dispersar partículas de fármaco hidrofóbico (classe II do SCB) e ii) aumentar a taxa de permeação dos fluidos aquosos no conteúdo da cápsula. Subsequentemente, ocorre a minimização de aglomerados de partículas em contato com o meio de dissolução e, por fim, a otimização da área superficial efetiva ${ }^{26}$. Baseado nisto, sugere-se que a Farmácia $\mathrm{C}$ tenha empregado concentrações $(\% \mathrm{~m} / \mathrm{m})$ semelhantes à Farmácia B.
Sabe-se que os desvios de qualidade mais frequentes nos medicamentos magistrais podem ser atribuídos à quantidade de fármaco insuficiente, composição inadequada de excipiente e procedimentos tanto de homogeneização quanto de distribuição não efetivos $^{9,10}$. Tais irregularidades levam a grandes variações na UC e influenciam negativamente o desempenho biofarmacotécnico de formas farmacêuticas sólidas orais, impactando especialmente a dissolução.

Vale destacar que a dispensação apropriada de cápsulas manipuladas de atorvastatina pode minimizar riscos clínicos relacionados ao tratamento da dislipidemia e reduzir o custo do mesmo.

O presente trabalho demonstrou a importância do monitoramento do processo magistral aplicado bimestralmente a diferentes formulações, visto que o ensaio de determinação de peso de cápsulas de ATC realizado lote a lote pelo controle de qualidade nos estabelecimentos magistrais não correspondeu verdadeiramente à quantidade declarada de ATC, em \%, referente à determinação de UC. Soma-se a isto, o fato de uma das farmácias ter o produto reprovado no teste de dissolução.

Em conclusão, pode-se dizer que é necessário que todo medicamento, seja industrializado ou manipulado, apresente a quantidade de fármaco declarada e a cedência de acordo com o tempo especificado. Por fim, amplia-se a relevância da constante avaliação da qualidade de formas farmacêuticas trazendo à tona as dificuldades do setor magistral, bem como evidencia-se a efetividade dos critérios de aprovação empregados até o momento.

\section{AGRADECIMENTOS}

Os autores agradecem o apoio financeiro da FAPEMIG e da PROPE-UFSJ.

\section{CONFLITO DE INTERESSE}

Os autores declaram não haver qualquer potencial conflito de interesse que possa interferir na imparcialidade deste trabalho científico.

\section{REFERÊNCIAS}

1. Faludi, AA, Izar MCO, Saraiva JFK, Chacra APM, Bianco HT, Afiune Neto A. et al. Atualização da Diretriz Brasileira de Dislipidemias e Prevenção da Aterosclerose - 2017. Arq Bras Cardiol. 
2017;109(2Supl.1):1-76.

DOI: http://dx.doi.org/10.5935/abc.20170121

2. Khan FN, Dehghan MHG. Enhanced bioavailability of atorvastatin calcium from stabilized gastric resident formulation. AAPS Pharm Sci Tech. 2011;12(4):1077-86. DOI: http://dx.doi.org/10.1208/s12249-011-9673-3

3. Relação Nacional de Medicamentos Essenciais: Rename 2020 [Internet]. Ministério da Saúde, Secretaria de Ciência, Tecnologia, Inovação e Insumos Estratégicos em Saúde, Departamento de Assistência Farmacêutica e Insumos Estratégicos. Brasília: Ministério da Saúde, 2020. 217 p. Acesso em: 8 Mar 2020. Disponível em: http://bvsms.saude.gov.br/bvs/publicacoes/relac ao medicamentos rename 2020.pdf

4. Moffat AC, Osselton MD, Widdop B. (Ed.). Clarke's analysis of drugs and poisons: in pharmaceuticals, body fluids and postmortem material. 3.ed. London: Pharmaceutical Press; 2011. v.2.

5. British Pharmacopoeia: BP 2012: Monographs: A-I. London: The Stationery Office, 2012. v.1. p.190-193.

6. Pfizer wins patent extension on cholesterol drug. Associated Press. 6 Jan 2009. Acesso em: 20 Ago 2019. Disponível em: http://www.nj.com/business/index.ssf/2009/01/ pfizer wins patent extension o.html

7. Pereira NF, Oliveira CMRCM. Análise do teor de captopril em farmácia de manipulação no município de Brumado. Rev Mult Psic. 2017;11(38):408-18.

https://doi.org/10.14295/idonline.v11i38.906

8. Souza KJ, Aléssio PV, Gomes AJPS. Desenvolvimento de excipiente específico para cápsulas de nifedipina preparadas magistralmente: parte I. Rev Ciênc Farm Básica Apl. 2009;30(3): 257-61.

9. Baracat MM, Montanher CLS, Kubacki AC, Martinez RM, Zonta GAN, Duarte JC. et al. Avaliação da qualidade de formulações manipuladas e industrializadas de sinvastatina. Lat Am J Pharm. 2009;28(3):427-32.
10. Markman BEO, Rosa PCP, Koschtschak MRW. Avaliação da qualidade de cápsulas de sinvastatina de farmácias magistrais. Rev Saúde Pública. 2010;(44)6:1055-62. DOI: https://doi.org/10.1590/S0034$\underline{89102010000600010}$

11. Leal AS, Melo FPS, Gomes TCB, Santana AS, Cunha TH, Saiki M. Avaliação preliminar da qualidade da fluoxetina comercializada por farmácias de manipulação em Belo Horizonte/MG. Vigil Sanit debate 2017;5(1):7683. DOI: https://doi.org/10.22239/2317$\underline{269 x .00810}$

12. Meireles BL, Grossi EB, Gonçalves PJ, Valle Verona ARV, Enes TB, Santos DMSS et al Divinópolis/MG - Implantação de serviços clínicos farmacêuticos em uma unidade da Estratégia Saúde da Família: uma parceria entre universidade e Secretaria de Saúde. Experiências Exitosas de Farmacêuticos no SUS. 2015;3(3):3643.

13. Resolução RDC № 67 , de 08 de Outubro de 2007. Boas Práticas de Manipulação de Preparações Magistrais e Oficinais para Uso Humano em Farmácias. Acesso em: 20 Ago 2019. Disponível

em: http://189.28.128.100/dab/docs/legislacao/resol ucao67 $08 \quad 10 \quad 07 . p d f$

14. Resolução RDC № 87 , de 21 de Novembro de 2008. Altera o Regulamento Técnico sobre Boas Práticas de Manipulação em Farmácias. Acesso em: 20 Ago 2019. Disponível em: http://bvsms.saude.gov.br/bvs/saudelegis/anvisa L2008/res0087 2111 2008.html

15. Narasaiah VL, Jimidi B, Goli V, Kanakam VB. Enhancement of dissolution rate of atorvastatin calcium using solid dispersions by dropping method. Int J Pharm Tech Res. 2011;3(2):652-59.

16. Atorvastatin Calcium Tablets. Revision Bulletin. The United States Pharmacopoeia 2018: USP 41: The National Formulary: NF 36. Rock Ville: United States Pharmacopoeia Convention. Acesso em: 20 Ago 2019. Disponível em: https://www.uspnf.com/sites/default/files/usp p df/EN/USPNF/revisions/atorvastatin-calciumtabs-rb-notice.pdf 
17. Aulton ME, Taylor KMG. Aulton: Delineamento de formas farmacêuticas. 4.ed. Rio de Janeiro: Elsevier; 2016.

18. Rowe RC, Sheskey PJ, Cook WG, Fenton ME (Eds.). Handbook of pharmaceutical excipients. 7.ed. London: Pharmaceutical Press; 2012.

19. Brazilian farmacopeia National Form. Brazil. Ministry of Health: Agência Nacional de Vigilância Sanitária. 2.ed. Brasília: ANVISA, 2012. Acesso em: 5 Jul 2019. Disponível em: http://portal.anvisa.gov.br/documents/33832/25 9372/Formulario+NACIONAL+FARMACOPEIA+ING LES+com+alerta.pdf/056212ea-c30e-4f80-bf0e63a7fdf6c149

20. Farmacopeia Brasileira. v. 1. Agência Nacional de Vigilância Sanitária. Brasília: Agência Nacional de Vigilância Sanitária, 2019. 874 p. Acesso em: 3 Nov 2019. Disponível em: http://portal.anvisa.gov.br/documents/33832/25 9143/Volume+1+Pronto.pdf/4ff0dfe8-8a1d-46b984f7-7fa9673e1ee1

21. Dissolution methods. US Food and Drug FDA. Acesso em: 14 Jun 2019. Disponível em: https://www.accessdata.fda.gov/scripts/CDER/di ssolution/dsp SearchResults.cfm

22. Recomendações para realização de ensaios de dissolução para formas farmacêuticas sólidas orais de liberação imediata. Acesso em 10 Ago $2019 . \quad$ Disponível em: http://portal.anvisa.gov.br/documents/33836/35 3599/recomenda dissolucao2.pdf/46895c559e66-416b-acb6-713e8addf779

23. Jee RD. Infrared spectroscopy. In: Moffat AC, Osselton MD, Widdop B. (Eds.). Clarke's analysis of drugs and poisons: in pharmaceuticals, body fluids and postmortem material. 4.ed. London: Pharmaceutical Press; 2011. v.1. p.521-37.

24. Silverstein RM, Webster FX, Kiemle DJ. Identificação espectrométrica de compostos orgânicos. 7.ed. Rio de Janeiro: LTC; 2012.

25. Pavia DL. et al. Introdução à espectroscopia. 4.ed. São Paulo: Cengage Learning, 2010.

26. Banakar UV. Pharmaceutical dissolution testing. New York: Marcel Dekke;, 1992. https://doi.org/10.1201/b14198
27. Swarbrick J, Boylan JC. Encyclopedia of Pharmaceutical Technology. 2.ed. New York: Marcel Dekker; 2002. v.2.

28. Osorio JG, Muzzio FJ. Effect of powder flow proprieties on capsule filling weight uniformity. Drug Develop Ind Pharm. 2013;39(9):1464-75. DOI:

http://dx.doi.org/10.3109/03639045.2012.72822 $\underline{7}$

29. Crouter A, Briens L. The effect of moiusture on the flowability of pharmaceutical excipients. AAPS Pharm Sci Tech. 2014;15(1):65-74. DOI: http://dx.doi.org/10.1208/s12249-013-0036-0

30. Eugenio BG, Silva KC, Romano KL, Tavares HS, Gomes AJPS. Ampliação dos critérios físicos de aceitação de cápsulas obtidas pelo processo magistral. JAPHAC. 2020;7:3. 